\title{
MONSTROS NO DISCURSO (META)FICCIONAL ${ }^{1}$ \\ Monsters in the (meta)fictional discourse
}

\author{
Renato Miguel Basso* \\ Lovania Roehrig Teixeira
}

\begin{abstract}
RESUMO
Este artigo tem por objetivo apresentar duas abordagens semânticas encontradas na literatura que procuram dar conta do discurso sobre ficção, a Abordagem Tradicional e a Abordagem de Mudança de Contexto, e demonstrar em quais aspectos essas abordagens falham ao analisar sentenças sobre ficção quando há indexicais envolvidos. Por isso, na busca por um tratamento semântico mais abrangente, defende a Abordagem Monstro (Predelli, 2008) que propõe um controverso operador-monstro que manipula o mundo relevante para o contexto. Além disso, oferece uma extensão desse modelo para um caso não previsto em Predelli (2008) e por nenhuma abordagem clássica, que defende a atuação de operadores-monstro para dados do português brasileiro.
\end{abstract}

Palavras-chave: ficção; indexicais; operadores-monstro; semântica.

\begin{abstract}
This paper presents and criticizes two semantic approaches to the discourse about fiction: the Traditional Approach and the Context Change Approach. Although both these approaches predict the right truth-conditions to sentences about fiction, they both fail to
\end{abstract}

${ }^{1}$ Os autores agradecem a leitura atenta e as sugestões de Roberta Pires de Oliveira e Ana Lúcia Pessotto dos Santos e dos pareceristas anônimos, que em muito contribuíram para a clareza do texto. Todos os equívocos são de nossa responsabilidade.

* UFSC. 
do so when there are indexicals involved. We argue, following Predelli (2008), that a more accurate analysis is one that uses a controversial operator on contexts, i.e. a kaplanian monster. We also investigate a different case of talking about fiction, e.g. 'I think I could live close to her', and argue that this case is another piece of evidence in favor of a monstrous-based approach to the discourse about fiction.

Keywords: fiction; indexicals; monster operators; semantics.

A determinação das condições de verdade de sentenças sobre ficção ${ }^{2}$ é tema de um longo debate em filosofia, mas é uma questão bem mais timidamente tratada em linguística. Em princípio, essa situação não tem nada de anômalo, pois, enquanto aos filósofos cabem investigações não apenas sobre a linguagem, mas também sobre nossa condição epistemológica e sobre a metafísica envolvida na compreensão de sentenças ficcionais, aos linguistas fica o princípio de que, seja como forem usadas as palavras, em contextos "reais" ou "ficcionais", sua contribuição será sempre a mesma, ou seja, não importa sobre o que falamos, os mecanismos linguísticos envolvidos serão os mesmos.

Esse quadro simples é desafiado por sentenças ficcionais que envolvem indexicais ${ }^{3}$; se o desafio é claro para um filósofo, para um linguista o que interessa é que os indexicais em sentenças ficcionais alteram os mecanismos de interpretação das sentenças e dos indexicais envolvidos (ou pelos menos é isso que proporemos), o que faz com que sentenças ficcionais com indexicais se revistam de interesse para um linguista, notadamente para um semanticista.

Ao longo deste artigo, exporemos os principais problemas que cercam as sentenças ficcionais na seção 1, juntamente com as principais

2 Sentenças ficcionais são classificadas em três grupos: sentenças metaficcionais (proferimentos sobre ficção), sentenças ficcionais (sentenças proferidas dentro da ficção) e sentenças transficcionais (proferimentos sobre mais de uma ficção). Por simplicidade, utilizaremos a expressão "sentenças ficcionais" para os proferimentos sobre ficção como, por exemplo, comentários sobre um filme.

3 Usaremos indexicais ao invés de indiciais ou dêiticos. Reservaremos o termo "dêitico" para certos usos que fazemos de alguns itens linguísticos; o termo "indicial" remete a qualquer item que deve ser interpretado em relação a algum índice ou parâmetro; finalmente, "indexical" isola os termos que recebem valor semântico em função de um contexto, conforme definiremos abaixo. 
propostas de solução encontradas na literatura. Ainda na seção 1, apresentaremos brevemente a abordagem clássica de Kaplan (1989) para os indexicais. Na seção 2, explicitaremos a proposta feita por Predelli (2008) que envolve um tipo controverso de operador, um operador que atua sobre contextos, batizado por Kaplan (1989) de "monstro". Na seção 3, defenderemos a proposta de Predelli e a estenderemos para outros casos antes não previstos. Finalmente, encerraremos o texto com as considerações finais.

\section{SENTENÇAS FICCIONAIS, INDEXICAIS E AS ROTAS PERCORRIDAS}

Nesta seção apresentaremos duas abordagens para o tratamento de sentenças ficcionais, a Abordagem Tradicional (AT) e a Abordagem de Mudança de Contexto (AMC) (PREDELLI, 2008). Como nosso interesse é o comportamento dos indexicais em sentenças ficcionais, faremos também uma breve exposição sobre esses itens, apresentando a proposta clássica de Kaplan (1989).

Brevemente, nossa problemática pode ser descrita do seguinte modo: sentenças ficcionais (Fic) e sentenças não ficcionais ou fatuais (Fat) podem ter a mesma estrutura superficial, e mesmo assim ter valores de verdade distintos, por conta de relacionarem-se com diferentes situações. Por exemplo, uma sentença como (1)

(1) Tiradentes foi enforcado sob o sol de outono,

pode ser proferida como um comentário acerca do filme "Os Inconfidentes", de 1972, que acabamos de assistir. A partir dessa contextualização ficcional ela será considerada falsa, pois em Fic (ou seja, no filme "Os Inconfidentes") Tiradentes foi enforcado em um dia nublado e ventoso de outono. No entanto, se tomarmos os fatos históricos (Fat) essa sentença pode adquirir um valor de verdade diferente, pois, se tivermos informações históricas de que no dia em que Tiradentes foi enforcado o tempo estava ensolarado, a sentença em (1) será considerada verdadeira, diferentemente de Fic. Como 
então explicar que uma mesma sentença pode apresentar valores de verdade diferentes quando relacionada a Fat ou a Fic ${ }^{4}$ ? No aspecto semântico, em que as sentenças se diferenciam?

Tomaremos, neste artigo, a representação de proferimentos como pares da forma sentença-contexto (PREDELLI, 2008). Por conta disso, todas as abordagens apresentadas serão baseadas em conjecturas sobre pares da forma $<\mathrm{s}, \mathrm{c}>$, ou seja, sentença-contexto. Marcaremos a diferença entre contextos e mundos ficcionais (Fic) e não ficcionais (Fat) através de diacríticos diferenciados. Por exemplo, para referência ao ficcional será utilizado (' ) e para o não ficcional será utilizado (*); uma variável sem qualquer diacrítico se refere a um contexto ou mundo possível não especificado. Desse modo, x', x faz referência ao ficcional; $\mathrm{x}^{*}, \mathrm{x}$ refere-se ao não ficcional; e $\mathrm{x}$ refere-se a um contexto ou mundo qualquer, i.e., não especificado.

\subsection{AS EXPRESSÕES INDEXICAIS}

Kaplan (1989) propôs a primeira grande análise semântica para os indexicais, para isso ele muniu-se da semântica de mundos possíveis e do conceito de designador rígido de Kripke (1980). Sua teoria apresenta dois pilares: a teoria da referência direta e a divisão do significado dos indexicais em caráter e conteúdo. Baseado na ideia de designador rígido, Kaplan propõe que os indexicais sejam termos diretamente referenciais, ou seja, termos que se referem a um dado objeto sem nenhum tipo de mediação, e se referem a esse mesmo objeto em todos os mundos possíveis em que esse objeto existe e

4 Deve-se notar que o problema posto pelas sentenças ficcionais é, sob certos aspectos, semelhante aos problemas colocados por sentenças modais, no sentido de que, em ambos os casos, sentenças são avaliadas em mundos diferentes do mundo real (atual). Porém, há certas peculiaridades com relação às sentenças ficcionais; uma delas, como veremos adiante, é o que resulta de sua combinação com indexicais, e outra é que elas se combinam com operadores modais, o que pode indicar que elas funcionam através de mecanismos diferentes daqueles por detrás de sentenças modais. Para guardar as diferenças e marcar nosso discurso sobre sentenças ficcionais, usaremos, relativamente a essas sentenças, a expressão contexto ficcional. 
também nos mundos onde ele não existe.

Considere a sentença em (2), que possui o item indexical 'eu':

(2) Eu poderia estar morto.

se o falante do contexto c, o contexto de proferimento de (2), é Pelé, Pelé será tomado como o referente de 'eu' em todos os mundos possíveis e acessíveis a partir (do mundo) de c, mesmo em mundos em que Pelé não existe. Assim, a sentença (2) em c será considerada verdadeira se, e somente se, em algum mundo possível w, o indivíduo denotado por 'eu’ em c (Pelé) está morto. No entanto, mesmo se o falante de 'eu' não existir no mundo w, 'eu' ainda denota o mesmo indivíduo lá, pois a referência de 'eu' é estabelecida no contexto de proferimento e não no mundo de avaliação (w). A ideia, grosso modo, é que indexicais envolvem um contexto para determinar seu valor (seu referente) e proposições envolvem mundos possíveis ou circunstâncias de avaliação para determinar seu valor (verdadeiro ou falso).

Outro aspecto importante a notar sobre os indexicais está relacionado com sentenças verdadeiras a priori, como é o caso de (3):

\section{(3) Eu estou aqui.}

Não precisamos saber nada sobre o mundo para sabermos que (3) é sempre verdadeira. No entanto, se considerarmos (3) num contexto configurado como <Pelé, campo do Santos>, (3) diria algo como em (4), que é verdadeira somente se soubermos algo sobre o mundo, ou seja, é verdadeira somente a posteriori (i.e., é uma sentença contingente).

(4) Pelé está no campo do Santos.

Devido a esse comportamento especial, Kaplan propõe dois passos, o caráter e o conteúdo, para se chegar ao significado das expressões indexicais.

Num primeiro momento, uma vez que os indexicais são expressões que dependem do contexto de proferimento para determinar seus referentes, precisamos estabelecer um conjunto de coordenadas que formam o contexto. 
Tais coordenadas podem ser representadas através de uma ênupla como $<\mathrm{c}_{\mathrm{a}}$, $\mathrm{c}_{\mathrm{h}}, \mathrm{c}_{\mathrm{t}}, \mathrm{c}_{\mathrm{l}}, \mathrm{c}_{\mathrm{w}}>^{5}$, e a partir dela chegamos ao caráter e conteúdo das expressões indexicais. O caráter é uma função que toma como input um dado contexto e produz como output um conteúdo; podemos entender o caráter como o valor pré-proposicional de um enunciado num dado contexto. Por sua vez, o conteúdo toma como input um mundo possível e produz um valor semântico: o conteúdo é o valor proposicional. O quadro abaixo ilustra esse mecanismo.

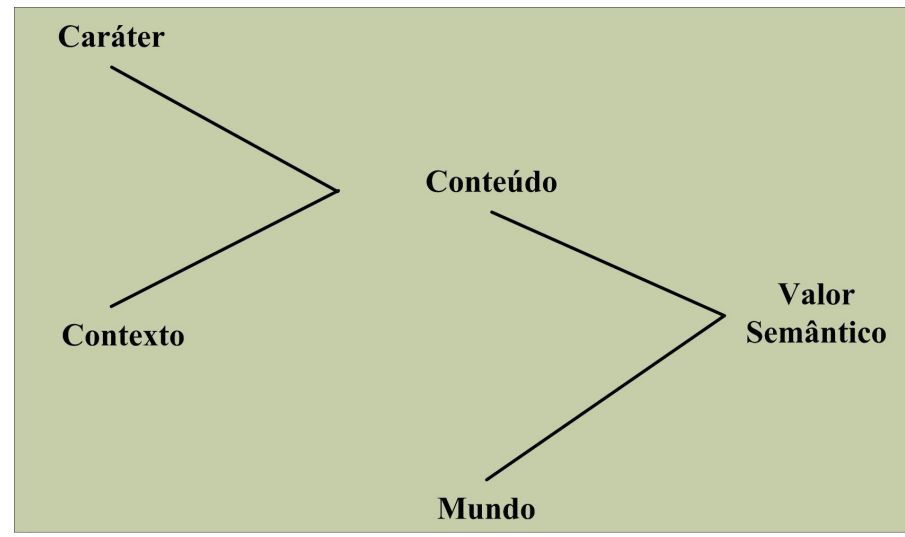

Figura 1: Esquema adaptado de Schlenker (2010, p. 7).

Tomando como exemplo a sentença em (3), e tendo como contexto $\mathrm{c}^{*}=\left\langle\mathrm{c}_{\mathrm{a}}=\right.$ Pelé, $\mathrm{c}_{\mathrm{h}}=$ Edinho, $\mathrm{c}_{\mathrm{t}}=2010, \mathrm{c}_{\mathrm{l}}=$ campo do Santos, $\left.\mathrm{c}_{\mathrm{w}}=\mathrm{w}^{* 6}\right\rangle$, chegaremos ao caráter e ao conteúdo:

5 Nessa notação, $c_{a}, c_{h}, c_{t}, c_{1}, c_{w}$ estão, respectivamente, por agente, ouvinte, tempo, local e mundo do contexto $c$, que, segundo Kaplan, é sempre o contexto de proferimento. Note que essa ênupla pode ser aumentada para incluir objetos presentes no contexto e assim dar conta do uso de demonstrativos.

${ }^{6} \mathrm{O}$ asterisco como sobrescrito representa o não ficcional. Assim, $\mathrm{w}^{*}$ faz referência ao mundo que habitamos (não ficcional), e c* faz referência ao contexto não ficcional, i.e., contexto de proferimento. Fazemos também a assunção simplificadora de que o mundo do contexto de proferimento $\left(\mathrm{c}_{\mathrm{w}}\right)$ é o mundo em que o falante está; dito de outra forma, se não houver necessidade do contrário, assumiremos que o mundo em que a sentença é avaliada (i.e., o mundo possível usado no conteúdo) é o mundo do contexto (i.e., $\mathrm{c}_{\mathrm{w}}$ ). Desse modo, avaliamos as condições de verdade de uma sentença, em princípio, em relação a um contexto e em relação ao mundo desse contexto. 
(3) $\mathrm{S}=$ Eu estou aqui

(3a) Caráter $(\mathrm{S})=\lambda \mathrm{c} \lambda \mathrm{w}\left[\mathrm{c}_{\mathrm{a}}\right.$ está em $\left.\mathrm{c}_{\mathrm{l}}\right]$

(3b) Conteúdo (S) = Caráter (S) $\lambda c \quad \lambda w\left[\mathrm{c}_{\mathrm{a}}\right.$ está em $\left.\mathrm{C}_{\mathrm{l}}\right]$

Conteúdo $(\mathrm{S})=$ Caráter $(\mathrm{S}) \lambda \mathrm{c} \lambda \mathrm{w}\left[\mathrm{C}_{\mathrm{a}}\right.$ está em $\left.\mathrm{c}_{\mathrm{l}}\right]\left(\mathrm{c}^{*}\right)$

Conteúdo $(\mathrm{S})=\lambda w$ [Pelé está no campo do Santos]

Assim, em prosa, para a sentença em (3) teremos um caráter como uma função que toma o contexto e produz o conteúdo. o caráter da sentença em (3) é igual ao agente do contexto estar na localização do contexto. Para que saibamos o significado completo da sentença, falta definir o conteúdo, que é uma função que toma um mundo possível e produz um valor semântico assumimos que o mundo a ser considerado (salvo indicações do contrário, como a presença de modais) é o mundo do contexto $c_{w^{\prime}}$ que é $w^{*}$. Assim sendo, o conteúdo da sentença em (3) é o caráter (o agente do contexto estar na localização do contexto) em relação a um mundo possível, o que vai gerar um valor semântico (nesse caso, um valor de verdade), e assim temos o conteúdo de (3) como sendo o conjunto de mundos em que Pelé está no campo do Santos; essas são as condições de verdade da sentença (3), com relação ao contexto $<\mathrm{c}_{\mathrm{a}}=$ Pelé, $\mathrm{c}_{\mathrm{h}}=$ Edinho, $\mathrm{c}_{\mathrm{t}}=2010, \mathrm{c}_{\mathrm{l}}=$ campo do Santos, $\mathrm{c}_{\mathrm{w}}=\mathrm{w}^{*}>$.

De acordo com Kaplan (1989), operadores podem manipular o conteúdo de um indexical; esse é o caso de operadores modais, como 'possivelmente' e 'necessariamente'. Voltando ao nosso exemplo (3), temos algo como:

(3c) $\mathrm{S}=$ Necessariamente $e$ estou aqui

(3c) é verdadeira sse em todos os mundos possíveis w' acessíveis a partir de $\mathrm{c}_{\mathrm{w}}, \mathrm{c}_{\mathrm{a}}$ (o agente) está em $\mathrm{c}_{1}$ (o lugar do contexto). Ao alimentarmos (3c) com nosso contexto c*, o resultado é, grosso modo, 'Necessariamente, Pelé está no campo do Santos', uma sentença que é verdadeira se em todos os mundos possíveis w’ acessíveis a partir de $\mathrm{c}_{\mathrm{w}}$ Pelé está no campo do Santos. (3c), diferentemente de (3), e de acordo com nossa intuição, não é verdadeira $a$ priori. 
Ainda segundo o autor, não há operadores que atuam sobre o caráter de um indexical em língua natural: não há operadores que modifiquem o contexto de avaliação de um indexical. Por conta disso, para Kaplan, o contexto dos indexicais é sempre o contexto de proferimento. Ele aceita que em linguagem formal o operador-monstro (como denominou o operador capaz de modificar o contexto de avaliação de um indexical) pode ser construído e adicionado, mas nega sua existência nas línguas naturais. Kaplan (1989) faz essas afirmações somente com base em dados do inglês, o que restringe severamente sua visão acerca do fenômeno ${ }^{7}$. Teríamos assim apenas operadores modais, aqueles que, nos termos de Kaplan, têm escopo apenas sobre as circunstâncias de avaliação (mundos possíveis). De modo semelhante, também a maioria das análises de sentenças que dizem respeito ao discurso ficcional e não ficcional está baseada em operações sobre mundos possíveis. Contudo, Predelli (2008) fornece argumentos de que precisamos de monstros para uma análise semântica adequada de sentenças Fic.

\subsection{Sentenças ficcionais e a Abordagem Tradicional (AT)}

Lembramos ao leitor que é possível tratar proferimentos considerando-os pares sentença-contexto, $<\mathrm{s}, \mathrm{c}>$. Dado que se um par $<\mathrm{s}, \mathrm{c}>$ recebe valores de verdade diferentes com relação a Fic e Fat, devemos forçosamente concluir que se trata apenas superficialmente do mesmo par, pois de outro modo não podemos explicar a diferença em valor de verdade. Se Fic e Fat estão relacionados a pares $<\mathrm{s}, \mathrm{c}>$ diferentes, a diferença pode estar em $\mathrm{s}$ ou em $\mathrm{c}$, ou seja, ou as sentenças analisadas em Fic e Fat são diferentes, ou os contextos de avaliação são diferentes. A AT atribui as diferenças de valor de verdade entre Fic e Fat ao fato de que as sentenças mobilizadas são diferentes, e não o contexto de avaliação (que será o contexto do proferimento). O defensor mais importante e pioneiro dessa abordagem é David Lewis (1978, 1983). 2003, 2010).

${ }^{7}$ Contudo, operadores-monstros foram encontrados em várias línguas (SCHLENKER, 
A sentença Fic possuiria um operador em sua representação que podemos expressar informalmente como 'de acordo com a ficção'. Segundo Lewis (1978, p. 37-38):

Let us not take our descriptions of fictional characters at face value, but instead let us regard them as abbreviations for longer sentences beginning with an operator: "In suchand-such fiction..." such a phrase is an intensional operator that may be prefixed to a sentence $\varphi$ to form a new sentence. But then the prefixed operator may be dropped by way of abbreviation, leaving us with what sounds like the original sentence $\varphi$ but differs from it in sense $^{8}$.

Assim, a representação do proferimento Fic da sentença em (1) segundo a AT consiste num operador descrito de maneira informal como 'de acordo com o filme "Os Inconfidentes", que atua sobre (1), juntamente com o contexto $c^{*}$.

Esse operador é o FT, e assim o par sentença-contexto de Fic é representado como $<\mathrm{FT}(\mathrm{s}), \mathrm{c}^{*}>$, ao passo que o de Fat é simplesmente $<\mathrm{s}$, $c^{*}>$. FT seria um operador de dois lugares, cujo primeiro argumento é preenchido por uma expressão que indica a ficção relevante, e o segundo argumento, por uma fórmula que representa a sentença em tela. Dessa maneira, a sentença que faz parte do par sentença-contexto de Fic para a AT pode ser representada como em (1AT) $)^{9}$, tomando a sentença-base em (1):

(1) Tiradentes foi enforcado sob o sol de outono.

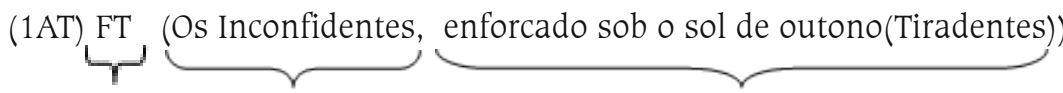

\section{Operador Ficção relevante Fórmula}

8 "Não tomemos nossas descrições de personagem ficcionais como elas aparecem, mas sim vamos tomá-las como abreviações de sentenças mais longas, com um operador: "Em tal-e-tal ficção..." Essa expressão é um operador intensional que pode ser prefixado a uma sentença $\varphi$ para formar uma nova sentença. Mas então esse operador prefixado pode ser removido por abreviação, nos deixando com o que parece com a sentença original $\varphi$, mas difere dela em sentido" (Tradução nossa).

9 Ao longo deste artigo, apresentaremos várias abordagens nas quais serão analisadas as mesmas sentenças. Assim, para que seja observado um padrão, as sentenças representacionais peculiares a cada abordagem terão em sua numeração as inicias da abordagem em questão. Por exemplo, (1AT) é a sentença (1) de acordo com a Abordagem Tradicional. 
FT deve ser visto como um operador intensional, cuja característica fundamental é a mudança do mundo possível das sentenças sobre as quais ele opera: FT determina a avaliação de uma sentença sob seu escopo a um mundo possível em particular ${ }^{10}$, em outras palavras, esse operador fixa o mundo determinado pela narrativa ficcional (seu primeiro argumento) como o mundo de avaliação da sentença representacional (seu segundo argumento). Formalmente, o operador FT pode ser representado como abaixo:

$[[\mathrm{FT}(\boldsymbol{\alpha}, \boldsymbol{\varphi})]]_{\mathrm{c}, \mathrm{w}}=\mathrm{V}$ sse $[[\varphi]]_{\mathrm{c}, \mathrm{w}^{\prime}}=\mathrm{V}$, onde $\mathrm{w}^{\prime}$ é o mundo determinado por $[[\alpha]] C, W$

Em prosa: $\varphi$, no escopo de FT, é verdadeira sse $\varphi$ é verdadeira no mundo w' de $\boldsymbol{\alpha}$, a narrativa ficcional relevante, estabelecida no contexto e mundo de proferimento $\mathrm{c}$.

Tal proposta traz a correta atribuição de valor semântico a Fic para (1AT), ou seja, o proferimento de (1AT) é V, sse a sentença 'Tiradentes foi enforcado sob o sol de outono' é avaliada como $\mathrm{V}$ com respeito ao mundo possível w', determinado pala narrativa ficcional "Os Inconfidentes". E, uma vez que no filme "Os Inconfidentes" o enforcamento de Tiradentes ocorreu sob tempo nublado, o resultado intuitivamente desejado é obtido: (1AT) é F em relação à w'; levando em conta, por fim, que o proferimento se deu no contexto $c^{*}, o$ que temos é que a sentença (1) é verdadeira em $c^{*}$ sse (1) é verdadeira no mundo determinado pela ficção; como (1) é falsa no mundo determinado pela ficção, ela também é falsa com relação a c*. Vejamos isso em passos:

(1) Tiradentes foi enforcado sob o sol de outono.

(1AT') <FT(Os Inconfidentes, enforcado sob o sol de outono(Tiradentes)),

10 Fazemos aqui outra assunção que não interfere diretamente em nosso propósito, que é considerar que se trata de um único mundo possível; na verdade, o ideal é dizer que FT determina um conjunto de mundos possíveis acessíveis a partir da ficção relevante e compatíveis com ele. Novamente, simplificaremos a discussão dizendo que há apenas um mundo ficcional relevante. 
$\mathrm{C}^{*}>=<\mathrm{FT}(\alpha, \mathrm{s}), \mathrm{C}^{*}>$

$\left(1 \mathrm{AT}{ }^{\prime \prime}\right) \mathrm{FT}(\boldsymbol{\alpha}, \mathrm{s})^{11}=\mathrm{V}$ sse $[[\mathrm{s}]]_{\mathrm{C}^{*}, \mathrm{w}^{\prime}}=\mathrm{V}$, onde $\mathrm{w}^{\prime}$ é o mundo determinado por $[[\alpha]]_{\mathrm{C}^{*}, \mathrm{w}^{*}}$

FT (Os Inconfidentes, enforcado sob o sol de outono(Tiradentes)) $=\mathrm{F}$ $\left(1 \mathrm{AT}^{\prime \prime \prime}\right)<\mathrm{FT}(\boldsymbol{\alpha}, \mathrm{s}), \mathrm{c}^{*}>=\mathrm{F}$

No entanto, a situação torna-se mais complexa quando há expressões indexicais envolvidas nos proferimentos Fic, principalmente, os que lidam com as coordenadas de mundo do contexto, como 'na realidade', que pode ser entendido como (PREDELLI, p. 283, 2008):

$[[\text { na realidade } \varphi]]_{\mathrm{c}, \mathrm{w}}=\mathrm{V}$ sse $[[\varphi]]_{\mathrm{c}, \mathrm{w}}=\mathrm{V}$, onde $\mathrm{w}$ é o mundo de $\mathrm{c}$, ou seja, $\mathrm{c}_{\mathrm{w}}$

Em prosa: 'na realidade $\varphi$ ' é verdadeiro em um mundo qualquer e em um contexto qualquer sse $\varphi$ é verdadeiro no mundo possível do contexto $\mathrm{c}\left(\mathrm{c}_{\mathrm{w}}\right)$, i.e., $\mathrm{w}=\mathrm{c}_{\mathrm{w}}$.

Considere o proferimento em (5), feito por alguém ao sair do cinema depois de assistir ao filme "Os Inconfidentes". Considere também que esse proferimento é falso de acordo com o filme ${ }^{12}$, e verdadeiro no mundo não ficcional, de acordo com a historiografia recente.

(5) embora Tiradentes fosse um homem sem posses e que buscava a igualdade de direitos, na realidade era muito rico e atuava como agiota.

Não ficção: Tiradentes era um homem rico.

Ficção: Tiradentes era um homem pobre.

Imagine que o filme deixa algumas pistas de que Tiradentes pudesse ser rico, ou seja, imagine que mesmo que Tiradentes não fosse mostrado

11 Estamos levando em conta o contexto de proferimento c* e o mundo $\mathrm{w}^{*}$.

12 No filme "Os Inconfidentes", Tiradentes é retratado como um mártir em busca da igualdade social e da redução de impostos que a coroa portuguesa impunha sobre o Brasil. No entanto, de acordo com documentos encontrados recentemente, Tiradentes era um homem muito rico e usava seu dinheiro na atividade de agiotagem; cf. $<$ http://www.istoe.com.br/reportagens/65363_CORRUPCAO+NA+INCONFIDENCIA+MINEIRA > . 
como alguém que tivesse bens e que lutava pela igualdade de direitos, há indícios no filme de que Tiradentes pudesse ser rico. Numa situação como essa, é possível que duas pessoas discutam sobre se Tiradentes no filme era de fato rico ou pobre. A sentença (5a) abaixo, ilustra justamente uma situação como essa:

(5a) [embora Tiradentes fosse um homem sem posses e que buscava a igualdade de direitos] Fic, [na realidade era muito rico e atuava como agiota] $\mathrm{Fic}=\mathrm{F}$.

Por sua vez, a sentença (5b) representa uma comparação entre o que é visto no filme e o não ficcional.

(5b) [embora Tiradentes fosse um homem sem posses e que buscava a igualdade de direitos] Fic, [na realidade era muito rico e atuava como agiota] Fat $=$ V.

O problema é capturar a interpretação (5a), que faz com que "na realidade' não considere o mundo do proferimento e sim o mundo ficcional. Dadas as definições de FT e de 'na realidade', teremos o seguinte: "na realidade (Tiradentes era muito rico e atuava como agiota)' é verdadeiro no mundo da ficção (segundo o operador FT), sse 'Tiradentes era muito rico e atuava como agiota' é verdadeiro no mundo do contexto (segundo o indexical 'na realidade'); portanto a sentença (5) só tem a interpretação (5b) de acordo com a $\mathrm{AT}^{13}$.

Tendo em vista essas duas leituras possíveis, a AT não possui os recursos necessários para que o proferimento em (5), na interpretação (5a),

13 Condições de verdade de (5AT): o proferimento, no contexto $c^{*}$, de (5AT) é verdadeiro sse [[Os Inconfidentes, na realidade (ser muito rico (Tiradentes))]] $\mathrm{c}^{*}, \mathrm{w}^{\prime}=\mathrm{V}$, onde $\mathrm{w}^{\prime}$ é o mundo possível determinado pela ficção "Os Inconfidentes", e ainda de acordo com a leitura da expressão indexical 'na realidade', sse [[ser muito rico (Tiradentes)] ]c*, w" $=\mathrm{V}$, onde $\mathrm{w}^{\prime \prime}=\mathrm{c}_{\mathrm{w}}$ (que assumimos que é $\mathrm{w}^{*}$, o mundo em que o falante faz o proferimento da sentença). 
Receba uma análise adequada ${ }^{14}$. O problema com a AT surge quando as sentenças Fic vêm acompanhadas de certos indexicais, como, por exemplo, 'na realidade' que afeta a coordenada mundo do contexto ${ }^{15}$.

\subsection{SENTENÇAS FICCIONAIS E A ABORDAGEM MUdANÇA DE CONTEXTO (AMC)}

Na AT observamos que a diferença entre Fic e Fat está justamente na sentença representacional do par $<\mathrm{s}, \mathrm{c}>$. Vimos também que quando combinados com certos indexicais a AT gera resultados incorretos. Diante disso, talvez seja o caso de abandonar a sentença s como foco das diferenças entre Fic e Fat e partir para a ideia de que devemos mudar o contexto no par $<\mathrm{s}, \mathrm{c}>^{16}$. E assim nasce a Abordagem de Mudança de Contexto que sugere que a representação de Fic deva incluir um contexto c' e um mundo possível w', que é o mundo do contexto determinado por "Os Inconfidentes" (PREDELLI, 1997; REIMER, 2005).

É importante notar que, de acordo com a AMC, uma vez que o contexto semanticamente relevante é alcançado no nível representacional, não é necessário que se apele a operadores não expressos, tais como

14 Lewis (1970, p. 22) tenta salvar a AT dos resultados inadequados da interação com 'na realidade' e sugere que nem sempre 'na realidade' possui essa leitura de indexicalidade. 0 autor afirma que a expressão 'real' e seus derivados têm dois sentidos diferentes (estipuladamente ambígua) - o sentido primário e o secundário. o sentido primário seria o que temos em (5b) e 'na realidade' refere ao mundo do proferimento $\left(\mathrm{w}^{*}\right)$. O sentido secundário atuaria sobre um contexto qualquer e não necessariamente o de proferimento. No entanto, a sugestão de Lewis é problemática, pois é nada mais do que uma estipulação ad hoc.

15 A AT esbarra em problemas semelhantes ao considerar o item 'agora', pois, equivocadamente, apela ao contexto de proferimento $c^{*}$ para a fixação de todos os indexicais, e pode ser o caso que certos indexicais sejam fixados nos contextos ficcionais.

16 Schlenker $(2003,2010)$ e Predelli (2008) mostram que o contexto envolvido na representação de um proferimento pode não incluir algumas das coordenadas (agente, tempo, mundo, local) do proferimento $\left(c^{*}\right)$, e sim de outro contexto qualquer. Considere a sentença, reproduzida por uma secretária eletrônica: 'Eu não estou no momento. Por favor, deixe seu recado após o bip.' O ouvinte, ao escutar esse proferimento, não o interpretará levando em consideração as coordenadas do contexto $\mathrm{c}^{*}$, pois teríamos a seguinte paráfrase: 'a secretária eletrônica não está no momento'; ele vai interpretá-lo, levando em consideração o contexto c" em que Maria, por exemplo, fez a gravação, cuja paráfrase seria: 'Maria não está no momento'. Essa situação mostra que nem sempre o contexto de proferimento $\left(\mathrm{C}^{*}\right)$ determina as coordenadas relevantes para a avaliação de um enunciado. Kaplan (1989) chamou tais contextos de "contextos impróprios". 
aparecem nas sentenças representacionais da AT (i.e., FT). Por tudo isso, o elemento responsável por reproduzir as diferenças entre sentenças Fic e Fat será o contexto relevante, c’ ou c*, e a sentença em (1AMC) será a mesma para representar tanto Fat quanto Fic.

(1) Tiradentes foi enforcado sob o sol de outono.

(1AMC) enforcado sob o sol de outono (Tiradentes)

A partir disso, teremos para Fat o par $<(1 \mathrm{AMC})$, $\mathrm{c}^{*}>$ e para Fic o par $<(1 \mathrm{AMC}), c^{\prime}>$. Com essa modificação em relação à AT, a AMC produz a avaliação esperada para Fic, pois as condições de verdade para Fic são: $(1 \mathrm{AMC})$ é verdadeiro no contexto $\mathrm{c}^{\prime},[[(1 \mathrm{AMC})]]_{\mathrm{c}^{\prime}, \mathrm{w}^{\prime}}=\mathrm{V}$, em que $\mathrm{w}^{\prime}$ é o mundo possível do contexto c', determinado pelo filme "Os Inconfidentes" sse é o caso que Tiradentes foi enforcado sob o sol de outono no filme. Em outras palavras, o proferimento 'Tiradentes foi enforcado sob o sol de outono' é verdadeiro sse Tiradentes foi enforcado em um dia ensolarado no mundo e no contexto determinados pelo filme "Os Inconfidentes". Portanto, a sentença em (1AMC) recebe o valor semântico adequado, ou seja, é falsa, pois, de acordo com o filme, Tiradentes foi enforcado num dia nublado.

A AMC, além de obter resultados adequados para sentenças Fic, também produz resultados adequados para sentenças Fic que envolvem expressões indexicais, como em (5), na interpretação (5a), o que não era o caso para a AT. Assim sendo, vamos analisá-la de acordo com a AMC:

(5a) [embora Tiradentes fosse um homem sem posses e que buscava a igualdade de direitos] Fic, [na realidade era muito rico e atuava como agiota] $\mathrm{Fic}=\mathrm{F}$.

A representação do proferimento em (5a) envolve o contexto de "Os Inconfidentes", ou seja, o contexto c' e a sentença representacional.

(5AMC) na realidade (ser muito rico (Tiradentes))

$\left(5 \mathrm{AMC}^{\prime}\right)<$ na realidade (ser muito rico (Tiradentes)), $\mathrm{c}^{\prime}>=<$ na realidade(s), 
c'>

$[[\text { na realidade } \varphi]]_{\mathrm{c}, \mathrm{w}}=\mathrm{V}$ sse $[[\varphi]]_{\mathrm{c}, \mathrm{w}}=\mathrm{V}$, onde $\mathrm{w}$ é o mundo de $\mathrm{c}$, ou seja, $\mathrm{c}_{\mathrm{w}}$ (5AMC') na realidade (ser muito rico (Tiradentes) $)_{\text {c', } \mathrm{w}^{\prime \prime}}=\mathrm{V}$, onde $\mathrm{w}^{\prime \prime}$ é o mundo de C'

$\left(5 \mathrm{AMC}^{\prime \prime \prime}\right)<\mathrm{S}, \mathrm{C}^{\prime}>=\mathrm{F}$

Condições de verdade: <(5AMC), c'> é verdadeiro se e somente se 'na realidade (ser muito rico (Tiradentes)) ' c', w, V, onde w", é o mundo de c'. Como na ficção (c') Tiradentes é pobre, a sentença (5AMC) é falsa e chegamos à interpretação (5a) vista acima ${ }^{17}$.

Vimos que, no caso do proferimento Fic de (5), a AMC obteve resultados adequados à nossa intuição. No entanto, há algumas objeções à AMC, como a dificuldade de essa abordagem dar conta de proferimentos em que há uma espécie de operador explícito que identifica a narrativa ficcional relevante. Assim, o proferimento Fic de (1)

(1) Tiradentes foi enforcado sob o sol de outono

tem uma estrutura superficial igual na AT e na AMC. No entanto, esse aspecto se relaciona com a sentença representacional de Fic, pois, na AMC não há menção à narrativa ficcional relevante, ou seja, não há um operador da forma de FT (como na AT) que sinaliza a ficção relevante para a análise da sentença. Assim, a sentença representacional que forma o par com o contexto c' na AMC é simplesmente:

(1AMC) enforcado sob o sol de outono (Tiradentes).

Por isso, a mudança de contexto de c* para c' (e também de $\mathrm{c}_{\mathrm{w}^{*}}$ para

17 Pode parecer que 'na realidade' aqui tem um comportamento monstruoso por levar em conta um contexto que não é o de proferimento. Contudo, neste caso, o indexical 'na realidade' não é monstruoso, pois tem seu mundo determinado pelo contexto do par $<\mathrm{s}$, c' $>$. Para um indexical ser monstruoso, ele deve ser avaliado em um contexto diferente do especificado no par. 
$\mathrm{C}_{\mathrm{w}}$ ) acontece, então, sem nenhuma marcação linguística, em nenhum nível de representação. Se é assim, expressões como 'De acordo com "Os inconfidentes"' seriam estritamente redundantes: seu papel é mudar o contexto (e o mundo) de avaliação, mas, na AMC, tal mudança ocorre sem a intervenção de expressões desse tipo. Qual é então o papel de tais expressões na AMC? Uma sentença como (6), de acordo com a AMC, seria redundante, mas esse certamente não é o caso:

(6) De acordo com "Os Inconfidentes", Tiradentes foi enforcado sob o sol de outono.

Outro possível problema com a AMC relaciona-se também com a sentença (5) e com a interpretação (5b); nesse caso, a primeira sentença seria representada por $\left\langle\right.$ s, c' $>$ e a segunda (que traz 'na realidade') por $\left\langle\mathrm{s}, \mathrm{C}^{*}\right\rangle$, ou seja, numa mesma sequência haveria dois contextos envolvidos e nenhum tipo de marcação ou indicação sobre quais contextos estão sendo utilizados. À AMC cabe então uma explicação de como dar conta de várias mudanças consecutivas de contexto sem o apelo a qualquer tipo de operador ou operação.

Tais problemas na AMC motivam a busca por outras explicações para sentenças ficcionais.

\section{OPERADOR SOBRE CONTEXTOS - MONSTROS}

Certamente há diversas ordens de problemas (e méritos) com a AT e com a AMC, mas a busca por uma abordagem mais satisfatória para sentenças sobre ficção nos leva a considerar outra alternativa.

Predelli (2008) propõe um tratamento diferente aos indexicais presentes nas sentenças Fic, mas com isso desafia a abordagem de Kaplan (1989). Kaplan (1989) nega que existam itens que operam sobre o caráter de um indexical em línguas naturais, em outras palavras, nega que existam operadores que manipulem o contexto em que os indexicais são avaliados esse contexto deveria ser sempre o contexto de proferimento, que 
representamos como $\mathrm{C}^{*}$. No entanto, como já foi mostrado por Schlenker (2003, 2010) e outros, tais operadores-monstros, no domínio epistêmico, foram encontrados em línguas como o amárico e o ewe ${ }^{18}$. E, assim como Schlenker, no domínio epistêmico, Predelli, no domínio modal, argumenta que há monstros que atuam sobre o contexto de avaliação dos indexicais em sentenças Fic, e que uma abordagem para sentenças ficcionais baseada em operadores-monstros pode dar conta dos problemas que vimos nas seções anteriores.

A Abordagem Monstro (AM), proposta por Predelli (2008) para os discursos sobre Fic, faz uma mescla da ideia de operadores-monstros para o tratamento das expressões indexicais, de um lado, e da AT e AMC para o tratamento do discurso sobre Fic, de outro. Nela, Fic e Fat se diferenciam através da sentença s do par sentença-contexto, pois para Fat o par é da forma $<\mathrm{s}$, $\mathrm{C}^{*}>$ e para Fic $<\mathrm{FM}(\mathrm{s})$, $\mathrm{C}^{*}>$; e FM é nosso operador-monstromodal que atua sobre sentenças ficcionais, e que explicitaremos abaixo.

Predelli (2008) utiliza operadores-monstros no tratamento de sentenças Fic, pois eles são duplamente indexados e tal característica permite que eles se comportem de duas maneiras:

(i) diferentemente dos operadores intensionais, o operador M (monstro) afeta o contexto em que indexicais são avaliados. Para Predelli (2008) um operador M, desse tipo, é definido como:

$[[\mathrm{M} \varphi]]_{\mathrm{c}, \mathrm{w}}=\mathrm{V}$ sse $[[\varphi]]_{\mathrm{c}^{\prime}, \mathrm{w}}=\mathrm{V}$ para $\mathrm{K}\left(\mathrm{c}^{\prime}\right)$, onde $\mathrm{K}$ é alguma coordenada do contexto relevante

(ii) o operador M pode afetar o ponto de avaliação, assim "[...] the shifted context results in the abandonment of the possible world selected by the original context $\mathrm{c}$, and in its replacement with the possible world determined by appropriate fiction. ${ }^{19}$ " (PREDELLI, 2008, p. 292). Esse é definido como:

18 Amárico é a língua oficial da Etiópia e o ewe é uma língua falada por aproximadamente seis milhões de pessoas principalmente em Gana, Togo e Benim.

19 "[...] o contexto mudado resulta no abandono do mundo possível selecionado pelo contexto original c, e em sua substituição pelo mundo possível determinado pela ficção apropriada" (Tradução nossa). 
$\left[\left[M^{*} \varphi\right]\right]_{c, w}=V$ sse $[[\varphi]]_{c^{\prime}, w^{\prime}}=V$ para $K\left(c^{\prime}\right)$ e $J\left(w^{\prime}\right)$, onde $K$ é uma coordenada do contexto relevante e J é o mundo possível apropriado

A partir desses comportamentos do operador $M$, temos a seguinte definição do operador FM, que é o operador-monstro-modal de Predelli (2008) para sentenças Fic.

$[[F M(\alpha, \varphi)]]_{c, w}=V$ sse $[[\varphi]]_{c^{\prime}, w^{\prime}}=V$, onde $c^{\prime}$ é como $c$, exceto que $c_{w}=w^{\prime}$, e $w^{\prime}$ é o mundo possível determinado por $[[\alpha]]_{c, w}$

Desse modo, c seria algo como $<\mathrm{c}_{\mathrm{a}}, \mathrm{c}_{\mathrm{h}}, \mathrm{c}_{\mathrm{t}}, \mathrm{c}_{\mathrm{l}}, \mathrm{c}_{\mathrm{w}}>$ e c' algo como $<\mathrm{c}_{\mathrm{a}}$, $\mathrm{c}_{\mathrm{h}}, \mathrm{c}_{\mathrm{t}}, \mathrm{c}_{\mathrm{l}}, \mathrm{c}_{\mathrm{w}}>>$; o importante a notar é que c é diferente de $\mathrm{c}^{\prime}$ justamente por em $c_{w} \neq c^{\prime}{ }_{w}$; ou seja, c e c' são iguais em tudo, menos na coordenada de mundo, que em c é o mundo do proferimento e em c' é o mundo ficcional.

A solução de Predelli produz uma análise adequada do proferimento Fic em (1), feito na saída do cinema, pois na solução-monstro esse proferimento é avaliado como um par da forma $<\mathrm{FM}(\mathrm{s}), \mathrm{c}^{*}>$, no qual a sentença representacional é dada em (1AM).

(1) Tiradentes foi enforcado sob o sol de outono

(1AM') FM (Os Inconfidentes, enforcado sob o sol de outono (Tiradentes))

$\left(1 \mathrm{AM}{ }^{\prime \prime}\right)[[\mathrm{FM}(\alpha, \varphi)]]_{c, \mathrm{w}}=\mathrm{V}$ sse $[[\varphi]]_{c^{\prime}, \mathrm{w}^{\prime}}=\mathrm{V}$, onde c' é como $\mathrm{c}$, exceto que $\mathrm{c}_{\mathrm{w}}=\mathrm{w}^{\prime}$, e w'é o mundo possível determinado por $[[\alpha]]_{c, w}$

(1AM"') [[FM (Os Inconfidentes, enforcado sob o sol de outono $($ Tiradentes $)$ ) $]]_{\mathrm{c}^{*}, \mathrm{w}^{*}}=\mathrm{V}$ sse $[[\text { enforcado sob o sol de outono (Tiradentes) }]]_{\mathcal{C}^{\prime}, \mathrm{w}^{\prime}}$ $=\mathrm{V}$, onde $\mathrm{c}^{\prime}$ é como $\mathrm{c}^{*}$, exceto que $\mathrm{c}_{\mathrm{w}^{*}}=\mathrm{w}^{\prime}$, e $\mathrm{w}^{\prime}$ é o mundo possível determinado por [[Os Inconfidentes] $]_{\mathrm{C}^{*}, \mathrm{w}^{*}}$ $\left(1 \mathrm{AM}^{\prime \prime \prime}\right)<\mathrm{FM}(\mathrm{S}), \mathrm{C}^{*}>=\mathrm{F}$

Como no filme "Os Inconfidentes" Tiradentes foi enforcado num dia nublado, a sentença (1) Fic é avaliada na AM, corretamente, como falsa.

Observamos que a sentença representacional de Fic em (1AM) 
apresenta um operador sentencial, parafraseável como 'de acordo com "Os Inconfidentes". Assim, a solução-monstro não tem a restrição que a AMC tem, ou seja, ela é capaz de atuar de maneira satisfatória em proferimentos nos quais há locuções que indicam a narrativa ficcional relevante, como pode ser visto em (6). Isso ocorre uma vez que, na solução-monstro, a locução 'de acordo com "Os Inconfidentes" tem o papel de determinar o mundo possível ficcional relevante, já que o contexto do par é o contexto do proferimento, $c^{*}$.

(6) De acordo com "Os Inconfidentes", Tiradentes foi enforcado sob o sol de outono.

Além da solução proposta por Predelli (2008) ser adequada para a análise de casos simples de Fic, ela também se mostra capaz de produzir bons resultados nos casos em que as abordagens anteriores falharam. Por exemplo, quando no proferimento Fic há uma expressão indexical como 'na realidade', que pretende enfocar a falsidade do proferimento em (5), na interpretação (5a), que afirma que de acordo com "Os Inconfidentes" Tiradentes, personagem ficcional, era rico e atuava como agiota, teremos o proferimento Fic na solução-monstro representado por um par que contém o contexto de proferimento $\mathrm{c}^{*}$ e uma sentença, contendo o operador FM, como em (5AM).

(5a) [embora Tiradentes fosse um homem sem posses e que buscava a igualdade de direitos] Fic, [na realidade era muito rico e atuava como agiota] Fic $=$ F.

(5AM) FM (Os Inconfidentes, na realidade (ser muito rico (Tiradentes)))

E para o proferimento Fic (5) obtivemos as seguintes condições de verdade: (5AM) é verdadeiro no contexto não ficcional sse [[na realidade (ser muito rico $($ Tiradentes $))]]_{\mathrm{c}^{*}, \mathrm{w}^{*}}=\mathrm{V}$, ou seja, sse $[[(\text { ser muito rico }(\text { Tiradentes }))]]_{\mathrm{c}^{\prime}, \mathrm{w}^{\prime}}=$ $\mathrm{V}$. 
(5AM) FM (Os Inconfidentes, na realidade (ser muito rico (Tiradentes))) $\left(5 \mathrm{AM}^{\prime}\right)[[\mathrm{FM}(\boldsymbol{\alpha}, \varphi)]]_{\mathrm{c}, \mathrm{w}}=\mathrm{V}$ sse $[[\varphi]]_{\mathrm{c}^{\prime}, \mathrm{w}^{\prime}}=\mathrm{V}$, onde c' é como c, exceto que $\mathrm{c}_{\mathrm{w}}=\mathrm{w}^{\prime}$, e $\mathrm{w}^{\prime}$ é o mundo possível determinado por $[[\alpha]]_{\mathrm{c}, \mathrm{w}}$ (5AM") $[[\text { FM (Os Inconfidentes, na realidade (ser muito rico (Tiradentes))) }]]_{\mathrm{C}^{*} \text {, }}$ ${ }_{\mathrm{w}^{*}}=\mathrm{V}$ sse $[[\text { na realidade (ser muito rico (Tiradentes)) }]]_{\mathrm{c}^{\prime}, \mathrm{w}^{\prime}}=\mathrm{V}$, onde c' é como $\mathrm{c}^{*}$, exceto que $\mathrm{c}_{\mathrm{w}^{*}}=\mathrm{w}^{\prime}$, e $\mathrm{w}^{\prime}$ é o mundo possível determinado por [[Os Inconfidentes] $]_{\mathrm{C}^{*}, \mathrm{w}^{*}}$

(5AM"') $[[$ na realidade (ser muito rico (Tiradentes) $)]]_{\mathcal{C}^{\prime}, w^{\prime}}=\mathrm{V}$ sse $[[$ ser muito rico (Tiradentes) $]_{\mathrm{C}^{\prime}, \mathrm{w}^{\prime}}=\mathrm{V}$, onde $\mathrm{w}^{\prime}$ é o mundo de $\mathrm{C}$ $\left(5 \mathrm{AM}^{\prime \prime \prime \prime)} \mathrm{F}<\mathrm{M}(\mathrm{S}), \mathrm{C}^{*}>=\mathrm{F}\right.$

Dado o que sabemos do filme, a sentença (5AM) será interpretada como falsa, pois no filme Tiradentes é um homem pobre.

Abaixo apresentamos um quadro sinótico do que as diferentes abordagens dizem com relação à sentença (5):

\begin{tabular}{|c|c|c|}
\hline $\mathrm{AM}$ & AT & $A M C$ \\
\hline (5a) $<\mathrm{FM}(\mathrm{s}), \mathrm{c}^{*}>$; $<\mathrm{FM}(\mathrm{s}), \mathrm{c}^{*}>$ & $(5 \mathrm{a})<\mathrm{FT}(\mathrm{s}), \mathrm{c}^{*}>;<\mathrm{FT}(\mathrm{s}), \mathrm{c}^{*}>$ & $(5 \mathrm{a})<\mathrm{s}, \mathrm{c}^{\prime}>;<\mathrm{s}, \mathrm{c}^{\prime}>$ \\
\hline$(5 b)<F M(s), c^{*}>;<s, c^{*}>$ & $(5 \mathrm{~b})<\mathrm{FT}(\mathrm{s}), \mathrm{c}^{*}>;<\mathrm{s}, \mathrm{c}^{*}>$ & $(5 b)<s, c^{\prime}>;<s, C^{*}>$ \\
\hline
\end{tabular}

A abordagem AT gera sem problemas a interpretação (5b), mas, devido à presença do indexical 'na realidade', a configuração (5a) resulta numa interpretação como a de (5b); a abordagem AMC gera, sem problemas, a interpretação (5a), mas carece de uma explicação independente de várias mudanças de contexto para dar conta da interpretação (5b). Por sua vez, a abordagem AM gera corretamente as duas interpretações.

\section{MAIS MONSTROS NA FICÇÃO}

Tendo em vista os casos analisados na seção anterior, e a obtenção de resultados intuitivamente desejados para os proferimentos Fic com e sem indexicais pela AM de Predelli (2008), nesta seção vamos defender a AM por meio de argumentos que não foram levantados até o momento e para um caso não previsto, e não tratado pelas abordagens anteriores. 
O caso que queremos trazer à tona pode ser ilustrado pela seguinte situação: imagine que, ao fim de uma peça de teatro, uma repórter entrevista duas atrizes, a Ana e a Joana, que interpretam, respectivamente, a Maria e a Mariana na peça, e pergunta à Ana:

(7) O que você acha que poderia ser feito para que a peça fosse melhor?

Ao que Ana responde:

(8) Eu acho que eu poderia morar mais perto dela (apontando para Joana).

A sentença (8), como veremos, coloca uma série de problemas bastante complexos, e uma análise completa da sentença foge ao escopo deste texto, mas um olhar um pouco mais detalhado sobre os indexicais nela envolvidos nos revela que há mais de um contexto sendo manipulado, o que leva a crer que há um operador-monstro atuando. Vejamos isso em passos.

Em primeiro lugar, vamos considerar o contexto $\mathrm{c}^{*}$, no qual temos Ana e Joana, e o contexto ficcional (da peça em questão) c', no qual temos Maria e Mariana. Assim sendo, uma análise correta da sentença (8) seria como (9) abaixo, com os contextos mobilizados explicitados em (9a):

(9) Ana acha que Maria poderia morar mais perto de Mariana.

(9a) $\mathrm{Eu}_{\mathrm{c}^{*}}$ acho que eu $\mathrm{u}_{\mathrm{c}^{\prime}}$ poderia morar mais perto dela $_{\mathrm{c}^{\prime}}$ (apontando para Joana $)^{20}$.

${ }^{20}$ Como bem notou um parecerista anômino, nossa interpretação preferencial é como

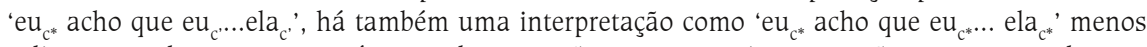
saliente ou relevante, que está em (10b), mas não temos uma interpretação como 'eu c' acho que $\mathrm{eu}_{\mathrm{c}^{\prime} . .}$ ela $_{\mathrm{c}^{\prime}}$ ' ou 'eu $\mathrm{c}^{\prime}$, acho que $\mathrm{eu}_{\mathrm{c}^{*} \ldots} \ldots \mathrm{ela}_{\mathrm{c}^{\prime}}$ '. Como argumenta Schlenker $(1999,2003,2010)$, a mudança do contexto de fixação de um indexical só ocorre após um verbo de atitude proposicional, o que impossibilitaria qualquer configuração que comece com 'eu ${ }_{c}$, acho que...'; por sua vez, Anand (2006), ao analisar operadores-monstros em diversas línguas, mostra que certas línguas apresentam algumas construções em que todos os indexicais sob um verbo de atitude proposicional se fixam em um contexto diferente daquele do proferimento, talvez similarmente ao consecutio temporum (como notou um dos pareceristas). Todas essas questões devem ser melhor entendidas e, uma vez que o forem, certamente iluminarão o fenômeno sob análise. 
Ou seja, a sentença em (8) é proferida, como resposta à questão, por uma atriz (Ana) direcionada à outra atriz (Joana); em (8) percebemos que a primeira ocorrência de 'eu' se refere à Ana, como falante do contexto c*, já o segundo 'eu' faz referência ao contexto ficcional e à personagem interpretada por Ana na peça (Maria), assim como o indexical 'dela' se refere à personagem que Joana interpreta (Mariana) e não à Joana como interlocutora de c*. Por conta disso, temos a seguinte situação: o indexical 'eu' com índice c* faz referência ao não ficcional, ou seja, a Fat; já 'eu' e 'dela' com índice c' fazem referência ao ficcional, a Fic ${ }^{21}$. Desse modo, uma paráfrase para (8) seria como (10a) e certamente não como $(10 b)^{22}$, que seria o resultado de avaliar todos os indexicais de (8) com relação ao contexto de proferimento:

(8) Eu acho que eu poderia morar mais perto dela (apontando para Joana). (10a) Ana acha que Maria poderia morar mais perto de Mariana. (10b) Ana acha que Ana poderia morar mais perto de Joana.

Em relação à sentença em (8), é importante ainda notar a presença do verbo de atitude proposicional 'achar que'; e, também, que a mudança de Fat para Fic dentro do proferimento não se relaciona exclusivamente à coordenada de mundo ou tempo do contexto, aspectos que vinham sendo analisados nas abordagens para Fic, e sim à coordenada agente do contexto e também ao objeto mostrado (para o caso do apontamento à Joana). De fundamental importância é, também, a presença do verbo modal ('poderia') e sua interação com outros elementos da sentença (notadamente com o operador-monstro que estamos prestes a propor).

${ }^{21}$ Como se trata de uma peça de teatro, podemos imaginar que no futuro quaisquer outras atrizes, a Sandra e a Vanessa, interpretem Maria e Mariana. Suponha então uma situação similar à de (8), na qual uma repórter pergunta à Sandra 'O que você acha que poderia ser feito para que a peça fosse melhor?'; suponha também que a resposta de Sandra seja como a de Ana, 'Eu acho que eu poderia morar mais perto dela (apontando para Vanessa)'. Nessa situação, a paráfrase mais adequada para a resposta de Sandra é 'Sandra acha que Maria poderia morar mais perto de Mariana' e não 'Sandra acha que Sandra poderia morar mais perto de Vanessa', o que mostra que o contexto mobilizado após 'acho que' é o contexto ficcional, no qual o agente do exemplo ilustrado será Maria.

22 Note, contudo, que (10b) não é impossível, mas é menos saliente/relevante na situação descrita. 
Nosso objetivo é apresentar uma solução para (8) nas linhas da proposta de Predelli (2008), ou seja, lançando mão de operadores-monstros. Antes, contudo, de partirmos para tal proposta, é interessante avaliarmos alternativas para a análise de (8), não apenas como reforço à AM, mas para exaurir as alternativas. Nossa busca começa na pragmática, com base nas implicaturas, em seguida passamos pela proposta de ambiguidade e, por último, consideramos usos atributivos dos indexicais.

\subsection{Alternativas AOS MONSTROS}

Visualizar as ocorrências do indexical 'eu' na sentença em (8) pelas lentes da pragmática significa apelar para possíveis implicaturas que atuem sobre seu uso. A implicatura que buscamos deveria ter como função mudar o contexto de fixação do referente do segundo 'eu' - note que é exatamente essa a função de operadores-monstros: mudar o contexto de fixação de indexicais. Como há motivações independentes para a existência de operadores-monstros, apelar para a pragmática apenas nesse caso não seria procedente; por outro lado, dizer que a pragmática pode dar conta de todos os casos de operadores-monstros também não procede, pois há línguas em que indexicais com comportamento monstruoso (i.e., que são avaliados em contexto diferente do contexto de proferimento) são marcados formalmente.

A próxima alternativa de análise dos indexicais utilizados no proferimento de (8) é a explicação por ambiguidade lexical; a palavra 'eu' teria dois significados diferentes: "falante ou agente do contexto" e, por exemplo, "a personagem que o falante do contexto interpreta". No entanto, apelar para ambiguidades é sempre uma manobra arriscada, ainda mais quando se trata de um item como um pronome. Além disso, novamente, a ambiguidade proposta resultaria exatamente no que um operador-monstro faria: mudar o contexto de fixação de 'eu'; haveria um 'eu*' e um 'eu'. Contudo, como há línguas nas quais tal marcação é feita formalmente, o apelo à ambiguidade de 'eu' fica enfraquecido e fere, também, a assunção de praticamente todos os semanticistas e filósofos; nas palavras de Chierchia "O 
significado de eu é sempre perfeitamente determinado: trata-se do falante." (2003, p. 65). Portanto, se considerarmos que o indexical 'eu' sempre se refere ao falante do contexto, e o que muda é justamente o contexto (através de um operador-monstro), podemos dar conta do caso em questão sem recorrer à ambiguidade. Assim sendo, cremos que devemos postular ambiguidade apenas se não houver alternativa possível para a análise de 'eu' em casos como o de (8) 23 .

Há, por último, a alternativa de que pelo menos um dos 'eu' em (8) seja usado atributivamente. Nesse caso, como o indexical que parece problemático é o segundo, poderíamos considerar que o primeiro indexical 'eu' tem uma leitura referencial, pois se refere ao falante do contexto de proferimento da sentença, um indivíduo particular determinado; já o segundo 'eu' está sendo usado atributivamente, ou seja, mostra "[...] the ability of pronominal indexicals to function quite similarly to definite descriptions ${ }^{24 "}$ (ABBOTT, 2010, p. 206). Os autores que mais detalhadamente desenvolveram abordagens para os usos descritivos de indexicais, como Nunberg (1993), Recanati (2005) e Elbourne (2008), propõem que a descrição que substitui o indexical está pelo "papel” (role) desempenhado pelo "alvo" do indexical, como os exemplos mostram:

(dito por alguém apontando para o Papa Bento XVI)

(11) Ele costumava ser italiano.

(11a) O Papa costumava ser italiano.

(dito por Dilma Roussef numa reunião de cúpula)

(12) A constituição me dá a palavra final.

(12a) A constituição dá a palavra final ao presidente.

${ }^{23}$ Note que numa saída como essa ecoa a proposta de Lewis para 'real' e derivados, que vimos com relação à AT na nota 14; e, obviamente, padece dos mesmos problemas, pois qualquer abordagem que não envolva ambiguidade é mais interessante do que uma que envolve, até mesmo porque é muito provável que em outras línguas a mesma sentença apresente a mesma leitura..

24 “[...] a habilidade de um pronome indexical de funcionar quase como descrições definidas" (Tradução nossa). 
Nas paráfrases (11a) e (12a) fica claro que a contribuição do indexical é de fato o papel desempenhado pelo seu "alvo" ou "índice" (cf. NUNBERG, 1993; ELBOURNE, 2008). Se aplicarmos os mesmos princípios para o exemplo (8), o resultado seria algo como:

(13) Ana acha que a atriz que interpreta Maria poderia morar mais perto da atriz que interpreta Mariana.

Através da paráfrase em (13), em que usamos descrições definidas no lugar dos indexicais, em primeiro lugar, não chegamos à interpretação obtida do proferimento em (8); em segundo lugar, a descrição definida, resultado do uso atributivo, dá a ideia de que 'eu' se refere a alguém, qualquer que seja, e, por isso, não tem um indivíduo específico como referente. No entanto, isso não é adequado, pois ao avaliar o indexical 'eu' com índice c' temos em mente um referente particular determinado, i.e., Maria. Logo, o uso atributivo como explicação alternativa para as duas ocorrências do indexical 'eu' na sentença em (8) também não se conforma à nossa intuição. Depois dessa confessadamente superficial consideração das alternativas, podemos voltar nossa atenção para uma solução dos indexicais em (8) que leva em conta a ideia de operadores-monstros.

\subsection{OUTRO OPERADOR-MONSTRO}

O operador que precisamos para dar conta da sentença (8) em muito se assemelha ao FM, porém, ao invés de mudar apenas a coordenada de mundo do contexto de fixação dos indexicais, deve mudar também outras coordenadas do contexto, como a de agente $\left(\mathrm{C}_{\mathrm{a}}\right)$ :

$\left[\left[\operatorname{FM}^{\#}(\alpha, \varphi)\right]\right]_{c, w}=V$ sse $[[\varphi]]_{c^{\prime}, w^{\prime}}=V$, onde c'é como $c$, exceto que (i) $c_{w}=$ $w^{\prime}$, e w' é o mundo possível determinado por $[[\alpha]]_{c, w}$, e (ii) $K\left(c^{\prime}\right)$, onde $K$ é alguma coordenada do contexto ficcional relevante (como $\mathrm{c}_{\mathrm{a}}, \mathrm{c}_{\mathrm{h}}, \mathrm{c}_{\mathrm{l}}$, etc.) 
Ou seja, com $\mathrm{FM}^{\#}$ o mundo do contexto é o mundo estabelecido pela ficção. Contudo, outras coordenadas contextuais podem ser também modificadas, o que chamamos de coordenadas relevantes, e nesses casos são as coordenadas do contexto ficcional que são utilizadas para a fixação do valor de indexicais. Obviamente, seria necessário definir, e talvez prever, minimamente quais seriam as coordenadas contextuais relevantes a serem alteradas nos proferimentos. No entanto, no presente texto nos concentraremos apenas nos indexicais 'eu' e 'ela' e deixaremos a determinação das coordenadas relevantes para um próximo estudo. A ideia é que é possível mudar quaisquer coordenadas do contexto (quando isso se fizer necessário).

Com o operador que propomos, é possível mobilizar o contexto ficcional c' para fixar o valor do segundo 'eu' e de 'dela' em (8), alcançando o resultado correto, que é, respectivamente, Maria e Mariana.

É necessário, contudo, resolver ainda uma questão: em (8) levamos em conta o contexto $c^{*}$ para a primeira ocorrência de 'eu' e o contexto ficcional c' para a segunda ocorrência de 'eu', como delimitar então o uso de um ou outro contexto? Seguindo a proposta de Schlenker (1999, 2003), a solução para tanto está justamente no verbo de atitude proposicional 'achar que'. De acordo com o autor, todo verbo desse tipo é uma espécie de fronteira entre contextos, eles podem indicar que o contexto de proferimento deve ser considerado, ou então que outros contextos devem ser levados em conta; em suma, verbos de atitude proposicional são potencialmente operadoresmonstros. Se isso estiver correto o que temos, grosso modo, é o seguinte:

(9a) $\mathrm{Eu}_{\mathrm{c}^{*}}$ acho que $\mathrm{eu}_{\mathrm{c}^{\prime}}$ poderia morar mais perto dela, (apontando para Joana).

$\lambda w[\lambda c[e u(c)$ acho-que $]] c^{*}\left[\lambda c F^{\#}(\alpha,[\operatorname{eu}(c) \ldots\right.$ ela(c) $\left.])\left(c^{\prime}\right)\right] ;$ em que c' é o contexto da ficção dado por $[[\alpha]]$.

Teríamos o contexto de proferimento $c^{*}$ para fixar os indexicais fora do escopo de $\mathrm{FM}^{\#}$, o verbo de atitude 'acho que' como delimitador de fronteira 
contextual e o operador-monstro $\mathrm{FM}^{\#}$ como o responsável por efetuar uma mudança contextual que fornecerá o contexto ficcional (c') no qual os indexicais sob seu escopo encontram seus valores, conforme explicitado abaixo:

(9AM') $\mathrm{FM}^{\#}(\alpha$, eu poderia morar mais perto dela).

$\left(9 A M^{\prime \prime}\right)\left[\left[\operatorname{FM}^{\#}(\alpha, \varphi)\right]\right]_{c, w}=V$ sse $[[\varphi]]_{c, w}{ }^{\prime}=V$, onde c' é como c, exceto que (i) $c_{w}=w^{\prime}$, e w'é o mundo possível determinado por $[[\alpha]]_{c, w}$, e (ii) $K\left(c^{\prime}\right)$, onde $\mathrm{K}$ é alguma coordenada do contexto ficcional relevante (como $\mathrm{C}_{\mathrm{a}}, \mathrm{c}_{\mathrm{h}}, \mathrm{C}_{\mathrm{l}}$, etc.)

(9AM"') $\left[\left[\mathrm{FM}^{\#}(\boldsymbol{\alpha} \text {, eu poderia morar mais perto dela }]\right]_{\mathrm{C}^{*}, \mathrm{w}^{*}}=\mathrm{V}\right.$ sse $[[\mathrm{eu}$ poderia morar mais perto dela] $]_{\mathrm{c}^{\prime}, \mathrm{w}^{\prime}}=\mathrm{V}$, onde $\mathrm{c}^{\prime}$ é como $\mathrm{c}^{*}$, exceto que $\mathrm{c}_{\mathrm{w}^{*}}=$ $\mathrm{w}^{\prime}$, e w' é o mundo possível determinado por $[[\alpha]]_{\mathrm{C}^{*}, w^{*}}$, e (ii) $K\left(c^{\prime}\right)$, onde $K$ é alguma coordenada do contexto ficcional relevante (como $c_{a}, c_{h}, c_{l}$, etc.); no caso, $\mathrm{c}_{\mathrm{a}}$ e os objetos a serem mostrados.

Se substituirmos as coordenadas de agente 25 do contexto c' e também os objetos e indivíduos a serem apontados em c', no trecho relevante, chegaremos à paráfrase em (10a), que é nossa interpretação da sentença (8), no que tange aos indexicais envolvidos depois do verbo de atitude proposicional 'achar que':

25 Conforme salientou um dos pareceristas anônimos, apesar de a paráfrase (10a) capturar nossa intuição sobre a interpretação da sentença (8), temos também uma forte intuição de que a Ana é a agente de ambos os 'eu' presentes na sentença (8). É necessário aqui separar com cuidado a noção kaplaniana de agente do contexto do referente do termo 'eu'. Como mostram Corazza et al. (2002) e Predelli (1998), em casos simples é possível igualar o agente do proferimento com o referente de 'eu'; contudo, em diversos outros casos, tal equivalência não é possível. Considere o seguinte exemplo: imagine o professor A em sua sala conversando com um aluno; a sala do professor A é de frente à sala do professor B. O professor B não está e vários alunos batem à sua porta para depois irem embora. Irritado, o professor A escreve um bilhete com os dizeres 'Eu não estou aqui agora' e o cola na sala do professor B. Para todos os efeitos práticos, o referente 'eu' do bilhete é o professor B; pense agora no ponto de vista do aluno, que viu o professor A escrevendo o bilhete; mesmo do seu ponto de vista, o referente do 'eu' será o professor B (pelo menos para manter a coerência e relevância da mensagem), contudo, o agente do 'eu' é certamente o professor A. Estamos, portanto, diante de um caso em que o agente e o referente de 'eu' não se equivalem, e algo semelhante vale para o nosso exemplo (8). 
(10a) Ana acha que Maria poderia morar mais perto de Mariana.

A história é bastante complexa até aqui, mas falta ainda computar o verbo modal 'poderia' e o predicado verbal 'morar'. Com relação a 'morar' é preciso notar que ele deve ser avaliado também em relação ao mundo ficcional, pois a mudança de localização das personagens se dá no mundo da ficção; portanto, a proposição <Maria, morar-mais-perto-de, Mariana> é avaliada nos mundos ficcionais. Note que isso já é feito pelo operadormonstro $\mathrm{FM}^{\#}$, ao garantir que o mundo a ser considerado é $\mathrm{w}^{\prime}\left(\mathrm{c}_{\mathrm{w}}=\mathrm{w}^{\prime}\right.$, e w' é o mundo possível determinado por $\left.[[\alpha]]_{c, w}\right)$.

A contribuição do verbo modal é bem mais complexa. Uma paráfrase razoável para o papel desse verbo seria: nos mundos possíveis que estão de acordo com o que acredita $\boldsymbol{o}$ falante, a peça seria melhor se a personagem Maria se mudasse para mais perto da personagem Mariana. O termo em negrito, "o falante", se refere a qual falante? Obviamente se refere à atriz que foi indagada, se refere à Ana. Podemos pensar que o modal tem uma fonte de ordenação teleológica, segundo a qual os mundos próximos do ideal (ou seja, mundos em que a peça é melhor) para o falante são mundos ficcionais acessíveis, a partir da ficção relevante, nos quais Maria mora mais perto de Mariana. Esse é um esboço do papel do modal 'poderia', mas uma análise completa desse item foge ao escopo do presente artigo. O ponto principal é mostrar que um operador-monstro-modal é requerido pela sentença (8) e reforçar os argumentos de que operadores-monstros são necessários para a descrição das línguas naturais. Além disso, se nossa análise estiver correta, o indexical 'eu' pode ser fixado em um contexto diferente do contexto de proferimento, e contextos ficcionais podem ser mobilizados para fixar diferentes tipos de indexicais.

\section{CONSIDERAÇÕES FinaIS}

Neste texto, apresentamos algumas das possibilidades encontradas na literatura para dar conta de certos tipos de sentenças ficcionais. Nosso 
interesse está centrado na investigação da combinação desse tipo de sentenças com indexicais. Diante de diversos problemas encontrados em duas abordagens comuns na literatura (a AT e a AMC), apresentamos a proposta de Predelli (2008) (a AM), que, mesmo baseada num controverso operador de mudança de contexto, conhecido como operador-monstro, apresenta resultados consoantes à nossa intuição. Feito isso, postulamos uma análise nas mesmas linhas da AM para dar conta de sentenças como 'Eu acho que eu poderia morar mais perto dela' dita por atores como comentários sobre uma dada ficção. Nossa proposta ainda deve ser trabalhada, mas cremos tratar-se de um avanço interessante tanto na defesa de operadores-monstros, quanto na investigação da semântica de sentenças ficcionais, e, se estiver correta, mostra que os contextos mobilizados para a fixação do valor de indexicais podem ser contextos ficcionais e também que um indexical como 'eu' pode ser fixado em contextos diferentes do contexto de proferimento.

\section{REFERÊNCIAS}

ABBOTT, B. Reference. New York: Oxford University Press, 2010.

ANAND, P. De De Se. Ph. D. Dissertation - Massachusetts Institute of Technology, USA, 2006.

CHIERCHIA, G. Semântica. Tradução de: Luiz A. Pagani, Lígia Negri, Rodolfo Ilari. São Paulo: Editora da UNICAMP, 2003.

CORAZZA, E.; FISH, B.; GORVETT, J. Who is I? Philosophical Studies, v. 107, p. 1-21, 2002.

ELBOURnE, P. D. Demonstratives as individual concepts. Linguistics and Philosophy, v. 31, p. 409- 466, 2008.

FREGE, G. Lógica e filosofia da linguagem. Tradução de: Paulo Alcoforado. São Paulo: Editora da Universidade de São Paulo, 1978.

KAPLAN, D. Demonstratives: An Essay on the Semantics, Logic, Metaphysics, and Epistemology of Demonstratives an Other Indexicals. In: ALMOG, J; PERRY, J; WETTSTEIN, H. (Ed.). Themes from Kaplan. New York: Oxford Univesity Press, 1989. p. 481-563.

KRIPKE, S. Naming and Necessity. Harvard University Press: USA, 1980. 
LEWIS, D. Anselm and Actuality. Nou, v. 4, p. 175-188, 1970 (reprinted in LEWIS, D. Philosophical Papers I, Oxford, Oxford Univesity Press, p. 10-25, 1983). . Truth in fiction. American Philosophical Quarterly, v. 15, p. 37-46, 1978.

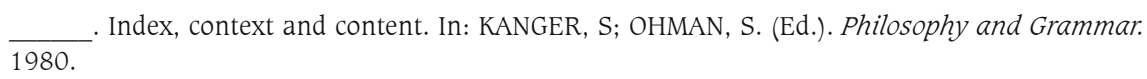

NUNBERG, G. Indexicality and deixis. Linguistics and Philosophy, v. 16, p. 01-43, 1993.

PREDELLI, S. Talk about Fiction. Erkenntnis, v. 46, p. 69-77, 1997. . I am not here now. Analysis, v. 58, p. 107-115, 1998. $\overline{297,2008 .}$

Modal Monsters and Talk about Fiction. Journal of Philosophical Logic, v. 37, p. 277-

RECANATI, F. Deixis and anaphora. In: SZABÓ, Z. G. (Ed.). Semantics vs. Pragmatics. Oxford: Clarendon Press, 2005. p. 286-316.

REIMER, M. The ellipsis account of fiction-talk. Studies in Linguistics and Philosophy, v. 81, p. 203-215, 2005.

SCHLENKER, P. A plea for monsters. Linguistics and Philosophy, Netherlands, Kluwer Academic Publishers, v. 26, p. 29-120, 2003.

. Indexicality and De Se Reports. Disponível em:

$\overline{<\mathrm{https}}$ ://files.nyu.edu/pds4/public/Schlenker-Indexicality\_and\_Del_Se.pdf > . Acesso em: $12 / 10 / 2010$

Propositional Attitudes and Indexicality: A Cross-Categorial Approach. Massachusetts Institute of Technology: USA, 1999.

Submetido em: 17/06/2011

Aceito em: 25/07/2011 